\title{
A Supplier Selection Model for End-of-Life Product Recovery: An Industry 4.0 Perspective
}

\author{
Ozden Tozanli, Poster Advisor: Prof. Elif Kongar
}

Department of Technology Management, School of Engineering, University of Bridgeport, Bridgeport, CT

Departments of Mechanical Engineering and Technology Management, School of Engineering, University of Bridgeport, Bridgeport, CT

\section{Introduction}

In order to sustain their long-term competitiveness, organizations are under constant pressure to strategically realign their businesses towards globalization and digitalization to adopt to changes in market dynamics.

This requires two major structural challenges to be addressed:

- How can manufacturers and consumers become active participants of environmentally responsible product disposal activities,

- How fast and efficiently manufacturers can respond to changing market and capital needs while preserving their environmental, economic, and social sustainability levels.

Industry 4.0 rises as a viable solution due to its ability to overcome these challenges and effectively respond to the increasing demand for mass customization. This concept is signified as a fully digitized future production and logistics system where all primary objects within a factory such as human, machine, and product as well as all partners within a supply chain such as supplier, manufacturer, logistics provider, and consumer are linked to one another.

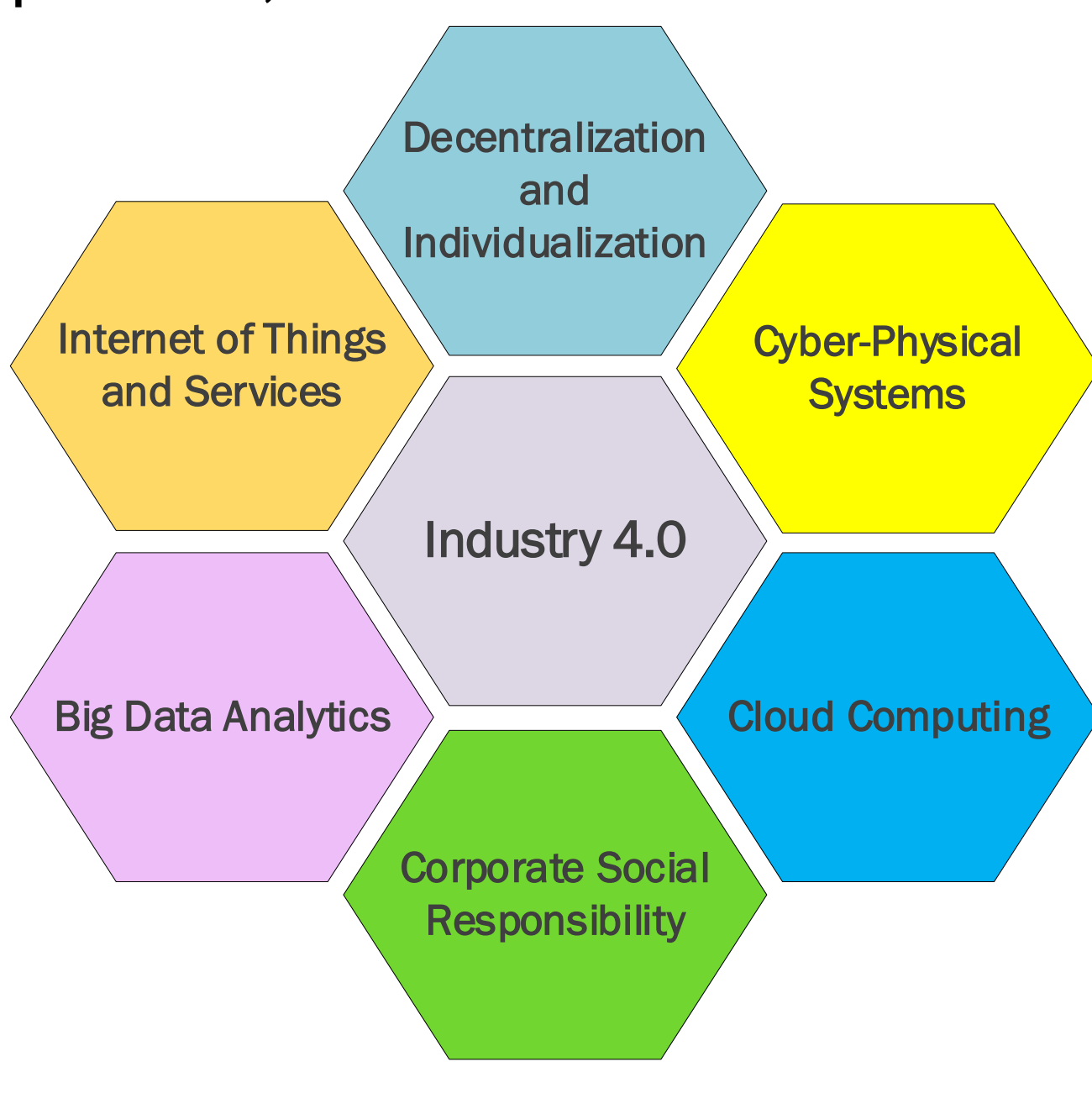

Value creation modules of Industry 4.0 leads to high degree of collaboration in value chains. Through Industry 4.0 , manufacturers could gain significant long-term advantages towards environmental economic and social sustainability.

Such degree of collaboration requires careful examination and evaluation of the performance and reliability of suppliers.

\section{Objectives}

Exhibit 1. Value creation modules of Industry 4.0

In end-of-life product recovery, the ability to monitor the quality levels of returned products remarkably affects to the quality of remanufactured products, increasing the revenue generated by material sales, and reducing the amount of products disposal.

The objective of this research is to evaluate suppliers based on their total cost, material sales revenue, total disposal weight, and the quality levels of products in the context of Industry 4.0.

The problem setting employs a new degree of control, design, transparency, and resource efficiency through:

\section{Internet-of-Things and Services \\ To keep track of individual products throughout their life cycles on a continuous basis via a series of embedded sensors.}

\section{Big data analytics}

To transform large volumes of data obtained from embedded sensors into valuable ubiquitous flow of information.

\section{Cloud computing}

To demonstrate a user-friendly interface of advanced digital platform which delivers a rapid real-time network connection.

\section{Cyber-Physical Systems}

To decode ongoing digital-to-physical and physical-to-digital cycles, therefore, maintaining the communication between these intelligent structures and humans.

Digital

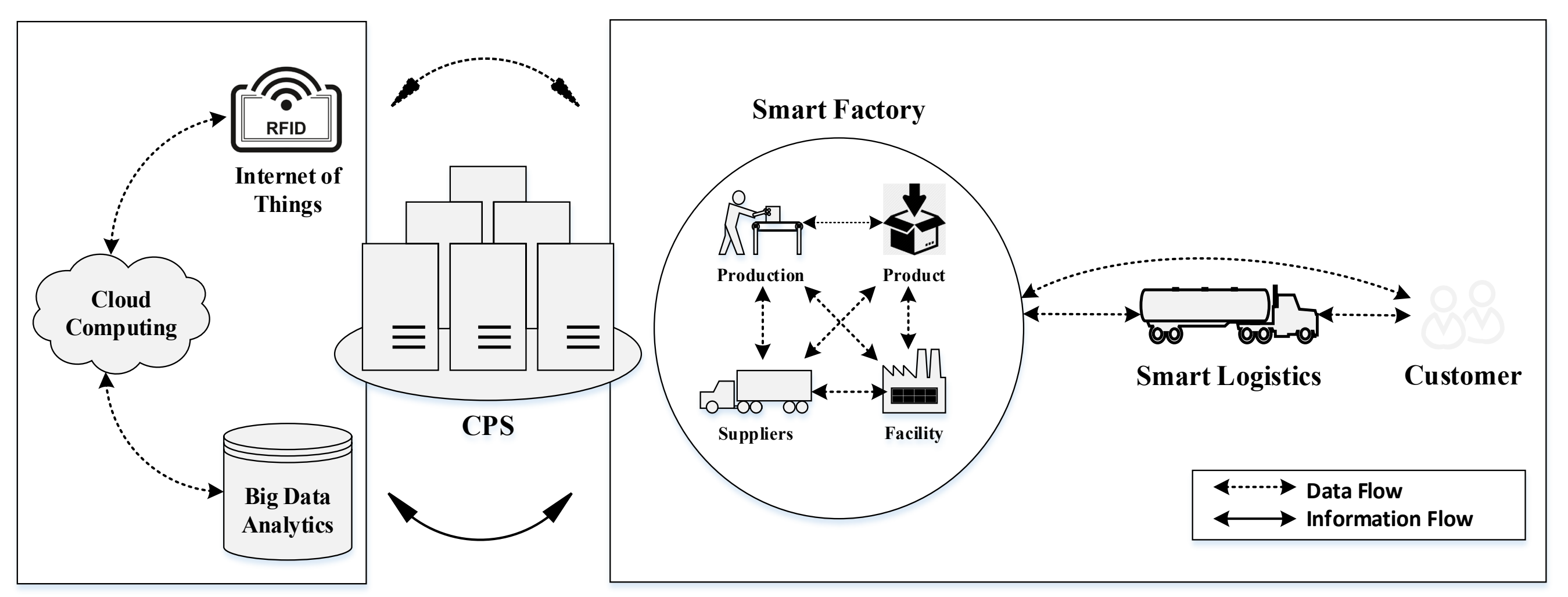

\section{Problem Design}

In this problem, digitization facilitates an unprecedented degree of surveillance and quality control along the point-to-point value chain from product design to end-of-life product recovery.

This allows real-time monitoring of material flows from raw material acquisition to end-of-life product recovery by enabling autonomous data exchange.

The manufacturer receives discarded products from multiple suppliers and communicate with returned devices to capture the data regarding their remaining useful life.

With dynamic data collection during the useful life time of products, the uncertainty surrounding disassembly operations are reduced significantly.

Final steps includes the manufacturer determining a recovery operation such as remanufacturing, reusing, recycling, or proper disposal based on the condition of each product.

\section{Methodology}

The problem is modeled as a multi-criteria decision making problem and is solved via a preemptive goal programming (GP) model for each supplier. The goals are:

- Minimizing the total cost,

- Maximizing the material sales revenue,

- Minimizing the total disposal weight,

- Maximizing the quality level.

When dealing with multiple objectives, GP formulates an objective function and a specific target value for each goal. Target values represent the minimum acceptance level for each goal. GP then aims at minimizing the overall deviation from each target value.

\section{Problem Statement}

- Two suppliers can supply 200 units of the same used product on a daily basis.

- The quality level of a product is determined by the remaining life of its components.

- The multiplicity, weight, recyclable percentage, probability of missing, unit recycling cost, unit disassembly cost, unit disposal cost, and unit holding cost of each component is deterministic.

\section{Results and Conclusions}
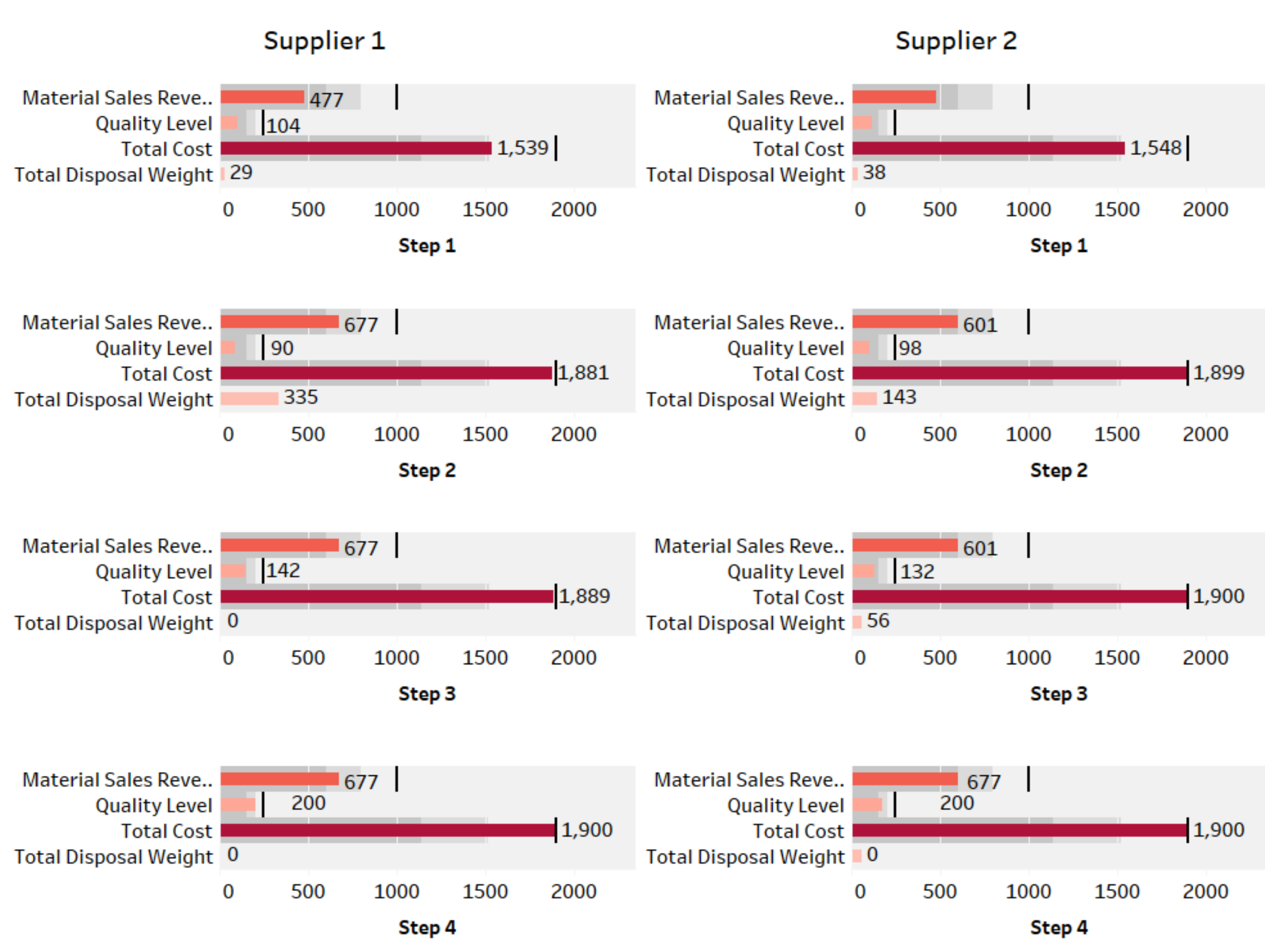

Exhibit 3. Target and Actual Performance Indicators of Each Supplier

While the goal of total cost is achieved by two suppliers, the goal of total disposal weight is only achieved by Supplier 1 . Based on the graphs, Supplier 1 demonstrates better performance than Supplier 2.

Overall, selecting the most appropriate suppliers based on quantitative data produces more efficient results leading to longterm partnerships.

References 